\title{
LOS RETOS DEL CUIDADO DE LAS PERSONAS CON DIABETES DURANTE EL ESTADO DE EMERGENCIA NACIONAL POR COVID-19 EN LIMA, PERÚ: RECOMENDACIONES PARA LA ATENCIÓN PRIMARIA
}

\author{
M. Amalia Pesantes (iD) ${ }^{1, a}$, Maria Lazo-Porras (id) ${ }^{1,2, b}$, Maria Kathia Cárdenas (id ${ }^{1, c}$, \\ Francisco Diez-Canseco (iD), d, Jessica Hanae Tanaka-Zafra (iD) 1,e , Rodrigo M.Carrillo-Larco (D) 1,3,f, \\ Antonio Bernabé-Ortiz (iD) 1,g, Germán Málaga (iD) ${ }^{1,4, h}$, J. Jaime Miranda (iD) 1,5,i \\ ${ }^{1}$ CRONICAS Centro de Excelencia en Enfermedades Crónicas, Universidad Peruana Cayetano Heredia, Lima, Perú. \\ 2 Departamento de Medicina Tropical y Humanitaria, Hospital Universitario de Ginebra y Universidad de Ginebra, \\ Ginebra, Suiza. \\ ${ }^{3}$ Department of Epidemiology and Biostatistics, School of Public Health, Imperial College London, Londres, Reino Unido. \\ ${ }^{4}$ Hospital Cayetano Heredia, Lima, Perú. \\ 5 The George Institute for Global Health, University of New South Wales, Sidney, Australia. \\ a Doctora en Antropología, magíster en Salud Pública; ${ }^{b}$ médica, magíster en Epidemiología; ${ }^{c}$ economista y magíster en \\ Economía de la Salud; ${ }^{\mathrm{d}}$ psicólogo, magíster en Salud Pública; ${ }^{\mathrm{e}}$ médica, magíster en Epidemiología; ${ }^{\mathrm{f}}$ médico; ${ }^{\mathrm{g}}$ médico, doctor \\ en Epidemiología; ${ }^{\mathrm{h}}$ médico, magíster en Medicina; ${ }^{\mathrm{i}}$ médico, doctor en Epidemiología.
}

\section{RESUMEN}

Las personas con diabetes mellitus tipo 2 infectadas por SARS-CoV-2 tienen mayores riesgos de desarrollar COVID-19 con complicaciones y de morir como consecuencia de ella. La diabetes es una condición crónica en la que se requiere continuidad de cuidados que implican un contacto con los establecimientos de salud, pues deben tener acceso regular a medicamentos, exámenes y citas con personal de salud. Esta continuidad de cuidados se ha visto afectada en el Perú a raíz de la declaratoria del estado de emergencia nacional, producto de la pandemia por la COVID-19 pues muchos establecimientos de salud han suspendido las consultas externas. Este artículo describe algunas estrategias que han desarrollado los diferentes proveedores de salud peruanos en el marco de la pandemia para proveer continuidad del cuidado a las personas con diabetes y finalmente brinda recomendaciones para que reciban los cuidados que necesitan a través del fortalecimiento del primer nivel de atención, como el punto de contacto más cercano con las personas con diabetes.

Palabras clave: Diabetes; Continuidad de Cuidados; COVID-19; Atención Primaria (Fuente: DeCs BIREME).

\section{HEALTHCARE CHALLENGES FOR PEOPLE WITH DIABETES DURING THE NATIONAL STATE OF EMERGENCY DUE TO COVID-19 IN LIMA, PERU: PRIMARY HEALTHCARE RECOMMENDATIONS}

\begin{abstract}
Citar como: Pesantes MA, Lazo-Porras M, Cardenas MK, Diez-Canseco F, Zafra-Tanaka JH, Carrillo-Larco RM, Bernabé-Ortiz A, Málaga G, Miranda JJ. Los retos del cuidado de las personas con diabetes durante el estado de emergencia nacional por la COVID-19 en Lima, Perú: recomendaciones para la atención primaria. Rev Peru Med Exp Salud Publica. 2020;37(3):541 6. doi: https://doi.org/10.17843/ rpmesp.2020.373.5980.
\end{abstract}

Correspondencia: María Amalia Pesantes; Avenida Armendáriz 445, Miraflores, Lima, Perú; maria.pesantes.v@upch.pe

\begin{abstract}
Patients diagnosed with type 2 diabetes mellitus, who then become infected with SARS-CoV-2, are at greater risk of developing complications from COVID-19, which may even lead to death. Diabetes is a chronic condition that requires continuous contact with healthcare facilities; therefore, this type of patients should have regular access to medicines, tests and appointments with healthcare personnel. In Peru, care and treatment continuity have been affected since the national state of emergency due to COVID-19 began; because many healthcare facilities suspended outpatient consultations. The strategies presented in this study were developed by different Peruvian health providers in the pandemic context to ensure care continuity for people with diabetes. This article provides recommendations to strengthen primary healthcare, because it is the first level of healthcare contact for patients with diabetes
\end{abstract}

Keywords: Diabetes; Continuity of Care; COVID-19; Primary Health Care (Source: MeSH NLM).

\section{INTRODUCCIÓN}

Las personas con diabetes mellitus tipo 2 que se contagian con el virus SARS-CoV-2 y que desarrollan la COVID-19 suelen tener más probabilidades de hospitalización, ingreso a las unidades de cuidados intensivos (UCI) y más comorbilidades que empeoran la gravedad de 
los síntomas ${ }^{(1,2)}$. En caso de que el paciente ingrese a la UCI, tener diabetes incrementa el riesgo de morir ${ }^{(3)}$. Los pacientes con enfermedades crónicas no transmisibles (ECNT), como la diabetes, requieren continuidad de cuidados, definida como el conjunto de cuidados que se debe garantizar y brindar de manera articulada para el bienestar del paciente y su familia ${ }^{(4)}$.

En el caso de la diabetes, esta continuidad de cuidados incluye usualmente el acceso regular a medicamentos, pruebas de laboratorio, citas con el personal de salud, facilidades para hacer actividad física, y llevar una dieta adecuada. Si bien estos cuidados son requeridos tanto para la diabetes mellitus tipo 1 como para la tipo 2, nuestro énfasis recae en esta última debido al incremento de casos en las últimas décadas y a cómo ha venido afectando mayoritariamente a la población económicamente activa ${ }^{(5)}$. En este artículo describimos los retos para garantizar esta atención en el marco del estado de emergencia nacional (EEN) debido a la pandemia en la ciudad de Lima, donde hasta mayo de 2020 se concentraban más del $60 \%$ de los casos y el $40 \%$ de los fallecidos del Perú ${ }^{(6)}$.

A fines de mayo del 2020, el Perú se convirtió en el segundo país con más casos y el tercero con más muertes por COVID-19 de América Latina ${ }^{(7)}$. Previamente, el 15 de marzo, el Gobierno peruano instauró el EEN estableciendo el aislamiento social obligatorio (cuarentena) para prevenir la propagación de la enfermedad mediante el Decreto de Urgencia 026-2020 que establece diversas medidas excepcionales y temporales para prevenir la propagación de la COVID-19 en el territorio nacional. El plazo del EEN ha sido extendido en numerosas ocasiones por el aumento de los casos, y a la fecha de elaboración de este artículo, se espera que culmine el 30 de junio. En este contexto, los recursos del fragmentado sistema de salud peruano se enfocaron en atender la mayor demanda generada por el rápido incremento de los casos de COVID-19, y su capacidad de respuesta fue rebasada en corto tiempo ${ }^{(8)}$, lo cual afectó los servicios disponibles para pacientes con otros problemas de salud ${ }^{(9)}$, entre los cuales se encuentran las personas con diabetes ${ }^{(10)}$.

Tomando como base la experiencia acumulada en diversos estudios realizados por CRONICAS Centro de Excelencia en Enfermedades Crónicas de la Universidad Peruana Cayetano Heredia, este artículo presenta aprendizajes que hacen visibles las limitaciones del sistema de salud para atender a las personas con diabetes antes de la aparición de la COVID-19; describe estrategias de algunos proveedores del sistema de salud en el marco de la pandemia para proveer continuidad del cuidado a las personas con diabetes y brinda recomendaciones para que reciban los cuidados que necesitan en el primer nivel de atención.

\section{Atención a los pacientes con diabetes antes de la COVID-19}

Nuestros estudios sobre las fortalezas y limitaciones del sistema de salud peruano para abordar las necesidades de los pacientes con ECNT, evidencian que en Lima hasta un tercio de los pacientes con diabetes habría recibido su diagnóstico al asistir a emergencias de un establecimiento de salud después de sufrir una complicación relacionada con su enfermedad ${ }^{(11)}$. También sabemos que las personas con ECNT que reciben atención del sistema de salud público enfrentan diferentes barreras para acceder a los medicamentos, conseguir citas para su seguimiento y control ${ }^{(12)}$, y para implementar recomendaciones a fin de manejar sus enfermedades crónicas ${ }^{(13)}$. Las normas existentes con relación a la atención de personas con diabetes reflejan una predominancia del manejo de estos casos centrada en especialistas, lo cual genera una mayor concentración de las atenciones en hospitales, mermando así las oportunidades para el control de diabetes en los centros de atención primaria ${ }^{(11)}$.

\section{Los servicios de salud para personas con diabetes durante el estado de emergencia nacional}

$\mathrm{Al}$ momento de la escritura de este artículo han pasado doce semanas desde que se inició la cuarentena obligatoria en el Perú. Es decir, durante este periodo de doce semanas la mayoría de las personas con ECNT, incluso la diabetes, no han recibido citas en los servicios de salud del Ministerio de Salud (MINSA) o del Seguro Social de Salud (EsSalud) debido a que las consultas externas no están habilitadas ${ }^{(14)}$. Asociaciones de pacientes han denunciado que debido al EEN por la COVID-19, existe cerca de un millón de personas con diabetes en el país afectados en cuanto a la reprogramación de citas y entregas de medicamentos ${ }^{(10)}$. Si bien la cuarentena impuesta varía entre países, en el Perú las restricciones no contemplan espacios ni la oportunidad para hacer actividad física. De hecho, recién en la semana 9 se permitió que los niños puedan salir con un adulto, por una hora, para hacer actividades recreativas en los parques. Por ejemplo, en países como Australia, si bien se promovió el distanciamiento físico durante la cuarentena, también hubo énfasis en preservar la actividad física, que es esencial para el manejo de muchas ECNT ${ }^{(15)}$.

En el Perú existen cuatro subsistemas de salud: MINSA, EsSalud, Fuerzas Armadas y Policiales, y establecimientos privados. En este artículo nos centramos en el MINSA y EsSalud que son los que atienden a la mayor parte de la población peruana. Como se observa en la Tabla 1, cada uno ofrece un tipo diferente de aseguramiento que le permite tener más o menos recursos para invertir en infraestructura, en recursos humanos y en tecnologías de la información. Todo esto sería útil en una situación de emergencia donde se evite que los pacientes con diabetes y otras enfermedades crónicas acudan a los establecimientos de salud.

Durante la cuarentena social obligatoria, cada uno de estos subsistemas del sistema de salud ha desarrollado sus propias estrategias para la atención a las personas con diversas enfermedades. Por ejemplo, la Dirección de Prevención y Control de Tuberculosis ha diseñado una serie de recomendaciones que permite que los familiares recojan 
Tabla 1. Subsistemas de salud, tipo y estrategias de aseguramiento

\begin{tabular}{|c|c|c|}
\hline Subsistema & Tipo de aseguramiento & Estrategias \\
\hline $\begin{array}{l}\text { Ministerio de Salud } \\
\text { (MINSA) }\end{array}$ & $\begin{array}{l}\text { El subsistema del Ministerio de Salud ofrece a través } \\
\text { del Seguro Integral de Salud (SIS) servicios de salud a la } \\
\text { población no asegurada a cambio del pago de una cuota } \\
\text { subsidiada }{ }^{(16)} \text {. Los afiliados al SIS pueden atenderse en la } \\
\text { red de establecimientos de los gobiernos regionales y del } \\
\text { MINSA }^{(17)} \text {. }\end{array}$ & $\begin{array}{l}\text { El MINSA ha impulsado en los últimos meses un amplio } \\
\text { uso de la telemedicina para la capacitación de personal } \\
\text { de salud del primer nivel de atención. Estas iniciativas } \\
\text { pueden continuarse y ampliarse a trabajadores de salud a } \\
\text { nivel nacional. Así como, promover que las Direcciones } \\
\text { Regionales de Salud exploren los métodos para que } \\
\text { los trabajadores de las regiones remotas accedan a la } \\
\text { información. }\end{array}$ \\
\hline $\begin{array}{l}\text { Seguro Social de Salud } \\
\text { (EsSalud) }\end{array}$ & $\begin{array}{l}\text { EsSalud ofrece servicios de salud a la población asalariada y a } \\
\text { sus familias en sus propias instalaciones }{ }^{(16)} \text {. Los empleadores } \\
\text { le pagan de manera obligatoria el } 9 \% \text { del salario del empleado } \\
\text { a EsSalud para que brinde esos servicios; además, EsSalud } \\
\text { cuenta también con un seguro potestativo para trabajadores } \\
\text { independientes o personas que no cuentan con SIS ni con el } \\
\text { seguro regular de EsSalud. }\end{array}$ & $\begin{array}{l}\text { EsSalud ha iniciado talleres de actividad física para } \\
\text { los miembros de sus Centros del Adulto Mayor, esto } \\
\text { puede promoverse para que pueda usarse masivamente, } \\
\text { ampliarlo a otros grupos etarios y usar la televisión para } \\
\text { que llegue a aquellos sin acceso a internet. Sobre todo, } \\
\text { considerando que los adultos mayores no tienen tanta } \\
\text { facilidad para el uso de tecnologías. }\end{array}$ \\
\hline
\end{tabular}

semanalmente el tratamiento para tuberculosis en el establecimiento de salud ${ }^{(18)}$. Sin embargo, hasta la fecha, no existe un plan oficial para el manejo de las ECNT durante el EEN, y permite que cada establecimiento desarrolle estrategias propias. Esto ocurre a pesar de que la prensa ha afirmado que el MINSA «viene propiciando que los pacientes que sufren hipertensión, diabetes o VIH solo acudan a los hospitales para el recojo de sus medicamentos, y no para sus consultas ambulatorias habituales» ${ }^{(19)}$. Una de las pocas estrategias que hemos identificado es la entrega de medicinas para pacientes crónicos en la casa o el recojo de estas en el sistema de EsSalud ${ }^{(20,21)}$. Lamentablemente, la fragmentación y la escasa articulación que existe en el sistema de salud peruano no permite que se implementen medidas para acceder oportunamente a las atenciones sanitarias en el contexto de la pandemia.

Con base en las conversaciones sostenidas con proveedores de algunos hospitales públicos de la ciudad de Lima, cada uno de los hospitales del MINSA estaría desarrollando una estrategia específica para brindar atención a los pacientes con diabetes. Lo común de estas estrategias es que buscarían mantener a los pacientes con ECNT alejados de las emergencias, que actualmente están superpobladas con personas sospechosas de tener la COVID-19. Por ejemplo, en el Hospital Nacional Cayetano Heredia se ha implementado un sistema de teleconsulta para atender pacientes con diabetes (Resolución Directoral 089-2020-HCH/DG). Sin embargo, en el Hospital Nacional Arzobispo Loayza se atiende a los pacientes con diabetes en el servicio de emergencias; si están estables se los deriva con el especialista para que les genere la receta y vayan a la farmacia para recibir sus medicamentos. Esta ruta expone a los pacientes a la COVID-19 y carga innecesariamente el área de emergencia. Estudios en otros países evidencian que la telemedicina es efectiva para promover un mejor autocuidado entre los adultos mayores con ECNT ${ }^{(22)}$, sin embargo, una revisión sistemática encontró que los niveles de health literacy ${ }^{(23)}$, que implica tener las competencias para acceder, comprender, evaluar y aplicar la información en salud, influyen en los beneficios de la telemedicina para el tratamiento de las ECNT. En el caso peruano habría que considerar, además, el limitado acceso a Internet de estos pacientes.

EsSalud, por su parte, ha suspendido todas las consultas ambulatorias, pero ha habilitado consultas usando telemedicina para pacientes de diferentes especialidades, incluyendo los de ECNT ${ }^{(24)}$. Actualmente se cuenta con la directiva «Telemedicina en pacientes con enfermedad crónica o continuador en EsSalud» orientada a realizar teleconsulta, telemonitoreo y teleorientación ${ }^{(25)}$. Entre los pacientes a los que se les está dando prioridad están aquellos con tuberculosis y VIH, así como los adultos mayores, muchos de ellos con diabetes, a quienes se brinda atención preferente para el recojo y la entrega de medicinas en sus viviendas a fin de que no acudan a los hospitales ${ }^{(26)}$.

\section{Implicancias de las restricciones de movilización para las personas con diabetes}

La reducción de la oferta en los servicios de salud para personas con ECNT y las restricciones de movilización tienen un impacto en la continuidad de cuidados de los pacientes con diabetes ${ }^{(9,10,27)}$, lo cual afecta su sobrevivencia, tal como sucede en otros países donde los excesos de muerte no son solo por la COVID-19, sino por otros factores, entre ellos las complicaciones por diabetes ${ }^{(28)}$. Esto puede deberse a distintas razones que requieren ser estudiadas. Es probable que el distanciamiento social obligatorio haya resquebrajado las redes de apoyo de algunas personas que viven solas, y también su posibilidad de adquirir los medicamentos que necesitan para su tratamiento. Además, la significativa reducción en la disponibilidad de transporte público afecta las posibilidades de acudir a un establecimiento de salud en caso de presentar complicaciones. 
Estudios en otros países han identificado que la incertidumbre de cuándo acudir a los servicios de emergencia por alguna complicación están teniendo un impacto negativo en aquellos con ECNT, pues no todos reconocen los signos de alarma de una complicación por diabetes ${ }^{(29)}$. Por ello, es de suma importancia prepararse para atender las diferentes ECNT una vez terminado el EEN, priorizando los casos que puedan derivar en complicaciones mayores, por ejemplo, pacientes con pie diabético.

Las restricciones de movilidad afectan las oportunidades de hacer actividad física, tal como lo demuestra un estudio en Italia ${ }^{(30)}$. Esto puede perjudicar a las personas con diabetes y otras ECNT. Para evitar el sedentarismo, Carvalho y Gois sugieren que las personas participen en las actividades del hogar (limpiar, lavar los platos o la ropa), jugar con los niños o con las mascotas además de caminar (cuando sea posible y necesario) para hacer las compras de la casa ${ }^{(31)}$. En el caso peruano, debido a que los mercados han sido foco de contagios, la recomendación más prudente es que se piense en estrategias para ejercitarse en casa, tomando en cuenta que muchos de los pacientes con ECNT son adultos mayores.

Debe además tomarse en cuenta que la cuarentena obligatoria está afectando significativamente el empleo y los ingresos de los hogares, especialmente de los más pobres y de quienes trabajan informalmente ${ }^{(32)}$. Esta reducción en la capacidad económica podría tener como resultado dificultades para adquirir alimentos saludables, asumir los costos de transporte y comprar medicamentos ${ }^{(10,33)}$, a pesar de que el Gobierno ha dispuesto la entrega de un bono económico para las familias de bajos recursos (Decreto de Urgencia 052-2020-EF).

\section{CONCLUSIONES}

La COVID-19 presenta grandes desafíos al fragmentado sistema de salud peruano, los cuales se reflejan tanto en las experiencias de las personas con una ECNT y en sus hogares, así como en la estructura de la organización de los servicios de salud para la ECNT. Este periodo podría también constituirse en una oportunidad para fortalecer el sistema de salud a fin de que esté más preparado para una mejor atención a las personas con ECNT durante la cuarentena y al finalizar esta. En este artículo hemos tomado como ejemplo la diabetes, a través de la cual hacemos notar que el primer reto es garantizar que las personas con ECNT reciban los servicios esenciales para sobrellevar una enfermedad crónica y prevenir complicaciones.

Para ello, el primer paso es reorientar el manejo de las ECNT hacia el primer nivel de atención ${ }^{(34)}$ para brindar la atención necesaria y evitar que las personas con diabetes y otras ECNT acudan a los hospitales durante y después de la cuarentena. Ello requiere trabajar en los cuatro pilares de la atención primaria para las personas con diabetes: accesibilidad y primer contacto, longitudinalidad, integralidad y coordinación ${ }^{(35)}$. Así, para fortalecer la atención primaria se requieren diferentes acciones que mencionamos a continuación:

1. Asignación de recursos materiales esenciales y la cantidad de recursos humanos adecuados para atender a personas con ECNT en puestos y centros de salud basados en datos epidemiológicos sobre la prevalencia de distintas ECNT y no en valores históricos que subestiman las demandas reales debido al subdiagnóstico.

2. Capacitación del personal de salud en el diagnóstico y manejo de la diabetes y otras ECNT, así como en el uso de tecnologías o estrategias que les permita ser el punto de primer contacto de la comunidad con el sistema de salud. Esto debe ir acompañado de normas orientadas a homogeneizar los cuidados para un escenario de limitada movilidad social y que establezcan una hoja de ruta clara para la provisión de servicios en los diferentes niveles de atención y entre los distintos proveedores de salud.

3. Garantizar que el personal de salud del primer nivel de atención puedan plantear sus dudas sobre el manejo de pacientes a especialistas mediante teleconsultas. Esta medida también contribuirá a brindar atención oportuna y a mejorar la confianza del propio personal de salud en sus habilidades para el manejo de ECNT.

4. Diseñar e implementar estrategias socialmente adecuadas para brindar cuidados a las personas con ECNT usando la tecnología (teleconsultas para ciertos grupos) u otras estrategias como anuncios televisivos y radiales que promuevan comportamientos saludables durante la cuarentena y después de esta. Asimismo, se pueden promover acciones comunitarias basadas en redes de soporte locales como los agentes comunitarios en salud.

5. Mejorar el acceso a la salud e integralidad de los servicios de salud . Las personas con diabetes deben tener garantizadas evaluaciones con un personal de salud (por telemedicina o presencial) y toma de muestra de sangre trimestralmente para la medición de hemoglobina glicosilada. Esto deberá estar acompañado de una evaluación y manejo de problemas de salud mental, acceso a consejería nutricional y de actividad física, acceso gratuito a medicamentos esenciales y el tamizaje de complicaciones crónicas. Estas estrategias tendrían que darse en el marco de visitas domiciliarias organizadas de manera articulada entre el Ministerio de Salud, el Ministerio de Desarrollo e Inclusión Social y las municipalidades.

Contribuciones de los autores: MAP, ML y MKC concibieron y diseñaron el artículo. MAP, ML, MKC, FDC, GM y JJM redactaron el artículo. JZT, RMCL, ABO y JJM hicieron la revisión crítica del artículo. MAP, ML, MKC, FDC, JZT, RMCL, ABO, GM y JJM aprobaron la versión final.

Financiamiento: JJM reconoce haber recibido financiamiento de Alliance for Health Policy and Systems Research (HQHSR1206660), the Bernard Lown Scholars in Cardiovascular Health Program at Harvard T.H. Chan School of Public Health (BLSCHP-1902), Bloomberg 
Philanthropies (via University of North Carolina at Chapel Hill School of Public Health), FONDECYT via CIENCIACTIVA/CONCYTEC, British Council, British Embassy and the Newton-Paulet Fund (223-2018, 2242018), DFID/MRC/Wellcome Global Health Trials (MR/M007405/1), Fogarty International Center (R21TW009982, D71TW010877), Grand Challenges Canada (0335-04), International Development Research Center Canada (IDRC 106887, 108167), Inter-American Institute for Global Change Research (IAI CRN3036), Medical Research Council (MR/P008984/1, MR/P024408/1, MR/P02386X/1), National Cancer Institute (1P20CA217231), National Heart, Lung and Blood

\section{REFERENCIAS BIBLIOGRÁFICAS}

1. Hussain A, Bhowmik B, Cristina do Vale Moreira N. COVID-19 and Diabetes: Knowledge in Progress. Diabetes Res Clin Pract. 2020;162:108142. doi: 10.1016/j.diares.2020.108142.

2. Gamble A, Pham Q, Goyal S, Cafazzo JA. The Challenges of COVID-19 for People Living With Diabetes: Considerations for Digital Health. JMIR Diabetes. 2020; 5: e19581. doi: 10.2196/19581.

3. Fang L, Karakiulakis G, Roth M. Are patients with hypertension and diabetes mellitus at increased risk for COVID-19 infection? Lancet Respir Med. 2020;8:e21. doi: 10.1016/S2213-2600(20)30116-8.

4. American Academy of Family Physycians [Internet] USA. AAFP; 2019. [citado el 6 de junio de 2020]. Continuity of Care, Definition of. Disponible en: https://www.aafp.org/about/policies/all/definition-care.html.

5. Carrillo-Larco RM, Bernabé-Ortiz A. Diabetes mellitus tipo 2 en Perú: una revisión sistemática sobre la prevalencia e incidencia en población general. Rev Peru Med Exp Salud Publica. 2019; 36,26-36. doi: 10.17843/rpmesp.2019.361.4027.

6. Ministerio de Salud [Internet] Lima: MINSA; 2020. [citado el 6 de mayo 2020]. Covid 19 en el Perú-Ministerio de Salud. Disponible en: https://covid19.minsa.gob.pe/sala_situacional.asp.

7. Collyns D. Peru's coronavirus response was 'right on time' - so why isn't it working? The Guardian [Internet]. 2020. [citado el 5 de junio 2020] Disponible en: https://www.theguardian.com/global-development/2020/may/20/peru-coronavirus-lockdown-new-cases.

8. Diario Gestión. Ciro Maguiña sobre el sistema de salud: "Hemos llegado a un límite, el colapso está ad portas". Gestión [Internet]. 2020. [citado el 25 de mayo 2020] Disponible en: https://gestion.pe/peru/coronavirus-peru-ciro-maguina-sobre-el-sistema-de-salud-hemos-llegado-a-un-limite-el-colapso-esta-ad-portas-covid-19-nndc-noticia/.

9. Salud con Lupa [Internet].Lima. 2020; Los otros pacientes. [citado el 8 de junio 2020] Disponible en: https://saludconlupa.com/series/los-otros-pacientes/.

10. Arana Conde R. COVID-19: Un millón de personas con diabetes se perjudican por falta de atención | Conexión Vida [Internet].2020. [citado el 28 de mayo 2020] Disponible en: https://conexionvida. net.pe/2020/04/18/covid-19-un-millon-de-personas-con-diabetesse-perjudican-por-falta-de-atencion/.

11. Cardenas M, Miranda JJ, Beran D. Delivery of Type 2 diabetes care in low- and middle-income countries: lessons from Lima, Peru. Diabet Med. 2016;33:752-60. doi: 10.1111/dme.13099.

12. Taype-Rondan A, Lazo-Porras M, Moscoso-Porras M, Moreano-Sáe$\mathrm{nz} \mathrm{M,} \mathrm{Miranda} \mathrm{JJ.} \mathrm{Inadequate} \mathrm{glycaemic} \mathrm{control} \mathrm{in} \mathrm{LMIC:} \mathrm{health}$ system failures in Peru. Br J Gen Pract. 2016;66: 197. doi: 10.3399/ bjgp16X684541.

13. Pesantes MA, Tetens A, Valle AD, Miranda JJ. "It is Not Easy Living with This Illness": A Syndemic Approach to Medication Adherence and Lifestyle Change among Low-income Diabetes Patients in Lima, Peru. Hum Organ. 2019; 78: 85-96. doi: 10.17730/0018-7259.78.1.85.

14. Barja L. Coronavirus: ¿Cómo afecta la pandemia de la COVID-19 a los pacientes diabéticos? rpp.pe [Internet] 2020. [citado el 10 de junio 2020] Disponible en: https://rpp.pe/vital/salud/coronavirus-como-afecta-la-pandemia-de-la-covid-19-a-los-pacientes-diabeticos-noticia-1260980.
Institute (HHSN268200900033C, 5U01HL114180, 1UM1HL134590), National Institute of Mental Health (1U19MH098780), Swiss National Science Foundation (40P740-160366), Wellcome (074833/Z/04/Z, 093541/Z/10/Z, 103994/Z/14/Z, 107435/Z/15/Z, 205177/Z/16/Z, 214185/Z/18/Z, 218743/Z/19/Z) and the World Diabetes Foundation (WDF15-1224). RMCL cuenta con financiamiento del Wellcome Trust International Training Fellowship (214185/Z/18/Z).

Conflictos de interés: Los autores declaran no tener ningún conflicto de interés.
15. Australian Government, Department of Health. Canberra: AGDH [Internet]; 2020 [citado el 10 de junio del 2020] Ongoing support during coronavirus (COVID-19). Disponible en: https://www.health.gov. au/news/health-alerts/novel-coronavirus-2019-ncov-health-alert/ ongoing-support-during-coronavirus-covid-19.

16. Cetrángulo O, Bertranou F, Casanova L, Casalí P. El Sistema de Salud del Perú: Situación actual y estrategias para orientar la extensión de la cobertura contributiva; 2013.

17. Alcalde-Rabanal JE, Lazo-González O, Nigenda G. Sistema de salud de Perú. Salud Publica Mex. 2011;53:s243-s254. Disponible en: http://www.scielo.org.mx/scielo.php?script=sci_arttext\&pi$\mathrm{d}=$ S0036-36342011000800019.

18. Ministerio de Salud. Recomendaciones para garantizar la administración del tratamiento antituberculosis en los establecimientos de salud [Internet]. Lima: MINSA; 2020 [citado el 1 de junio del 2020]. Disponible en: http://conamusa.org.pe/wp-content/uploads/2020/03/RECOMENDACIONES-TX.-ANTITB-Y-PAT-FRENTE-AL-COVID-19.-Final.pdf.

19. Andina [Internet]. Lima; 2020. [citado el 10 de Junio del 2020]. Covid-19: qué cuidados deben tener pacientes con diabetes o VIH. Disponible en: https://andina.pe/agencia/noticia-covid19-cuidados-deben-tener-pacientes-diabetes-o-vih-788767.aspx.

20. Seguro Social de Salud [Internet] Lima: Essalud; 2020. [citado el 10 de junio del 2020]. EsSalud continúa entrega de medicinas a pacientes de Consultorios de Atención Inmediata. Disponible en: http://noticias. essalud.gob.pe/?inno-noticia=essalud-continua-entrega-de-medicinas-a-pacientes-de-consultorios-de-atencion-inmediata.

21. Seguro Social de Salud [Internet] Lima: Essalud; 2020. [citado el 2 de junio del 2020]. Más de 200 mil pacientes crónicos de EsSalud recibirán sus medicinas en la farmacia de su distrito - Essalud. Disponible en: http://noticias.essalud.gob.pe/?inno-noticia=mas-de-200-mil-pacientes-cronicos-de-essalud-recibiran-sus-medicinas-en-la-farmacia-de-su-distrito.

22. Guo Y, Albright D. The effectiveness of telehealth on self-management for older adults with a chronic condition: A comprehensive narrative review of the literature. J Telemed Telecare. 2020;24:392-403. doi: 10.1177/1357633X17706285.

23. Parker S, Prince A, Thomas L, Song H, Milosevic D, Fort Harris M. et al. Electronic, mobile and telehealth tools for vulnerable patients with chronic disease: a systematic review and realist synthesis. BMJ Open.2018; 8: e019192. doi: 10.1136/bmjopen-2017-019192.

24. Seguro Social de Salud [Internet]. Lima: Essalud; 2020. [citado el 2 de junio del 2020]. EsSalud: pacientes crónicos reciben atención médica a través de telemedicina. Disponible en: Essalud http://noticias.essalud. gob.pe/?inno-noticia=essalud-pacientes-cronicos-reciben-atencion-medica-a-traves-de-telemedicina.

25. Seguro Social del Perú. Directiva de Gerencia General: 'Telemedicina en pacientes con enfermedad crónica o continuador en Essalud', 2020 [Internet]. Lima: Gerencia General. [citado el 9 de junio del 2020] Disponible en: http://www.essalud.gob.pe/wp-content/uploads/ RGG_632_GG_ESSALUD_2020.pdf. 
26. Seguro Social de Salud [Internet] Lima: Essalud; 2020. [citado el 8 de junio del 2020]. Consultorios de Atención Inmediata de EsSalud recibirán las 24 horas del día a pacientes en riesgo. Essalud http://noticias. essalud.gob.pe/?inno-noticia $=$ consultorios-de-atencion-inmediata-de-essalud-recibiran-las-24-horas-del-dia-a-pacientes-en-riesgo.

27. Hartmann-Boyce J, Morris E, Goyder C, Kinton J, Perring J, Nunan D, et al. Managing diabetes during the COVID-19 pandemic [Internet]. CEBM; 2020 [citado el 10 de mayo del 2020]. Disponible en: https://www.cebm.net/covid-19/managing-diabetes-during-the-covid-19-pandemic/.

28. The New York Times [Internet]. New York; 2020 [citado el 10 de mayo del 2020] . Lu D. There Has Been an Increase in Other Causes of Deaths, Not Just Coronavirus. Disponible en: https://www.nytimes. com/interactive/2020/06/01/us/coronavirus-deaths-new-york-newjersey.html? searchResultPosition $=1$.

29. Zhao J, Li H, Kung D, Fisher M, Shen Y, Liu R. Impact of the COVID-19 Epidemic on Stroke Care and Potential Solutions. Stroke. 2020;51(7):1996-2001. doi: 10.1161/STROKEAHA.120.030225.

30. Maugeri G, Castrogiovanni P, Battaglia G, Pippi R, D’Agata V, Palma $\mathrm{A}$, et al. The impact of physical activity on psychological health during
Covid-19 pandemic in Italy. Heliyon.2020; 6, e04315. doi: 10.1016/j. heliyon.2020.e04315.

31. Carvalho VO, Gois CO. COVID-19 pandemic and home-based physical activity. J Allergy Clin Immunol Pract .2020;S2213-2198(20)305109. doi: 10.1016/j.jaip.2020.05.018.

32. Gestión. [Internet]. Lima; 2020 [citado el 6 de mayo del 2020]. ¿Cómo perciben los hogares su situación financiera actual debido a la cuarentena? Disponible en: https://gestion.pe/fotogalerias/como-perciben-los-hogares-su-situacion-financiera-actual-debido-a-la-cuarentena-noticia/.

33. BBCNews Mundo [Internet]. London; 2020 [citado el 5 de mayo del 2020]. Gozzer S. El reto de poner en cuarentena a millones de personas en América Latina, una región en la que mucha gente sobrevive día a día. Disponible en: https://www.bbc.com/mundo/noticias-america-latina-51924224.

34. Beran D, Aebischer Perone S, Castellsague Perolini M, Chappuis F, Chopard P, Haller DM, et al. Beyond the virus: Ensuring continuity of care for people with diabetes during COVID-19. Prim Care Diabetes . 2020;S1751-9918(20)30199-6. doi: 10.1016/j.pcd.2020.05.014.

35. Vega Romero R, Acosta Ramírez N, Mosquera Méndez PA, Restrepo Vélez O. Atención primaria integral de salud: estrategia para la transformación del sistema de salud y el logro de la equidad en salud. Bogotá: Unión Gráfica; 2009. 